\title{
Reflets
}

Revue d'intervention sociale et communautaire

\section{Superviser des stagiaires dans une équipe communautaire de traitement intensif}

\section{Chantal Joly}

Volume 20, numéro 1, printemps 2014

La formation pratique : allier milieux de pratique et milieux

d'enseignement

URI : https://id.erudit.org/iderudit/1025801ar

DOI : https://doi.org/10.7202/1025801ar

Aller au sommaire du numéro

Éditeur(s)

Reflets, Revue d'intervention sociale et communautaire

ISSN

1203-4576 (imprimé)

1712-8498 (numérique)

Découvrir la revue

Citer cet article

Joly, C. (2014). Superviser des stagiaires dans une équipe communautaire de traitement intensif. Reflets, 20(1), 170-178. https://doi.org/10.7202/1025801ar 


\title{
Superviser des stagiaires dans une équipe communautaire de traitement intensif
}

\author{
Chantal Joly \\ Travailleuse sociale, \\ Équipe communautaire de traitement intensif, Ottawa
}

Bien qu'elle comporte des défis et qu'elle ajoute invariablement une charge à notre travail, la supervision de stagiaires demeure à mon avis une expérience enrichissante.

Travailleuse sociale dans l'Équipe communautaire de traitement intensif d'Ottawa (ÉCTI) depuis son implantation en 2000, j'ai eu certaines réticences quand on m'a demandé de superviser des stages. De nombreuses questions inondaient mes pensées : Suis-je prête? Mon bagage professionnel est-il suffisant? Comment est-ce que je définis mon rôle et comment transmettre mes connaissances? Ma personnalité sera-t-elle compatible avec celle de mon étudiante ou de mon étudiant? Mais le désir de vivre une telle expérience et la curiosité de m'y aventurer malgré l'inconnu m'ont poussée à relever ce défi. Il me fallait bien tenter l'expérience si je voulais des réponses à mes questions.

Le présent article porte donc sur mon expérience comme superviseure de stage. Je parlerai d'abord de l'historique et du fonctionnement de l'ÉCTI afin de préciser les particularités de ce milieu de stage. Je m'attarderai sur ma façon de conjuguer mon rôle de superviseure avec les défis reliés aux suivis communautaires. Enfin, je commenterai les apprentissages qui se vivent dans ce milieu et les liens entre la théorie et la pratique sur lesquels ils se fondent. 


\section{Historique des équipes communautaires de traitement intensif}

À la fin des années 1960 et tout au long des années 1970, à la suite du phénomène de désinstitutionnalisation, des transformations majeures s'opèrent dans le système de santé mentale. On vise à développer une approche permettant aux patients hospitalisés en psychiatrie de sortir de leur isolement et de se réinsérer dans la communauté.Toutefois, des recherches démontrent que le système de réseautage est insuffisant et manque d'efficacité pour assurer une réadaptation sociale adéquate. Afin de pallier ces lacunes, les professeurs Mary Ann Test et Leonard Stein ont mis sur pied le modèle Training in Community Living (TCL) au cours des années 1970 à Madison au Wisconsin. C'est ainsi qu'est née l'intervention en suivi communautaire qui vise à soutenir dans leur milieu de vie les personnes ayant des maladies mentales graves et persistantes (Test, 1998).

Depuis cet envol, ce domaine a évolué et fait place à un autre modèle, le Program of Assertive Community Treatment (PACT), connu en français sous le nom de "Programme de traitement intensif", lequel est géré par des ÉCTI. Les recherches démontrent son efficacité en ce qui a trait notamment à la diminution du temps passé à l'hôpital, à une meilleure autonomie résidentielle, à l'amélioration de l'état clinique et à une grande satisfaction de la part du client ou de sa famille (Test, 1998, p. 140). Le ministère de la Santé et des Soins de longue durée (MSSLD) de l'Ontario s'est donné un cadre conceptuel pour mieux clarifier les responsabilités et le mandat des ÉCTI.

L'une des responsabilités de ce ministère est d'assurer la gestion du système de santé provincial de l'Ontario. Il établit l'orientation stratégique de services incluant ceux en santé mentale. Jusqu'au $1^{\text {er }}$ avril 2007, le MSSLD finançait directement les hôpitaux afin de fournir des services de santé mentale aux adultes. Depuis, il finance les Réseaux locaux d'intégration des services de santé (RLISS) implantés dans chaque région de la 
province. Les RLISS se chargent de planifier et de financer la plupart des services communautaires et hospitaliers de santé mentale, incluant ceux de l'Hôpital Montfort. Le modèle PACT inspiré de Test et Stein a été une ressource indispensable pour développer les normes des ÉCTI, également connues sous d'autres vocables, anglophones et francophones. Parmi les plus utilisés en français, mentionnons : "Équipe de traitement communautaire actif », "Équipe de traitement dynamique » et «Équipe de suivi intensif dans la communauté " (Brouillette, l'Abbé et Tremblay, 2002, p.115). En Ontario, la première ÉCTI a été mise sur pied en 1990 (Lafave, De Sousa et Gerber, 1996). Le développement d'équipes a été influencé par des facteurs économiques, politiques et socioculturels (Brouillette, l'Abbé et Tremblay, 2002, p. 16) et le programme a connu une expansion nationale et internationale.

Le gouvernement de l'Ontario s'est appuyé sur le document Examen des meilleures pratiques de la réforme des soins de la santé mentale (Agence de la Santé publique du Canada, 2002) pour développer l'implantation des ÉCTI dans la province. Afin de maintenir une rigueur et une uniformité dans ces équipes, le gouvernement a publié deux documents complémentaires : Franchir les étapes: Cadre de prestations des services de santé mentale et des services de soutiens connexes (MSSLD, 1998a) et Standards for assertive community treatment Teams (MSSLD, 1998b). Par la suite, le gouvernement s'est inspiré des travaux d'Allness et de Knoedler qui ont publié en 2003 The National program standards for ACT teams. En Ontario, 79 ÉCTI ont été implantées en conformité avec les lignes directrices définissant les normes du programme.

\section{Fonctionnement}

L'ÉCTI est une équipe interdisciplinaire de professionnels de la santé mentale qui travaille avec des personnes ayant un trouble de santé mentale grave. Elle se compose de cliniciens issus de divers domaines (ergothérapie, soins infirmiers, travail social, intervention communautaire, soutien aux pairs, toxicomanie, psychologie, psychiatrie) qui se déplacent dans le milieu résidentiel de la 
personne et offrent des services de traitement, de réadaptation et de soutien. L'équipe comprend également une adjointe au programme, un poste administratif.

Les programmes de l'ÉCTI privilégient une approche globale ou holistique. Plus spécifiquement, ses membres sont appelés à « intervenir dans divers aspects fonctionnels de la personne " (Test, 1998). L'intervention peut porter tant sur le traitement - par exemple, la livraison de médicaments, la gestion des symptômes - que sur la recherche d'un travail, d'un logement ou de loisirs selon les objectifs individuels de chaque personne.

L'ÉCTI Ottawa est entièrement francophone et dessert la population de cette ville. Son mandat est de réhabiliter le client à partir d'objectifs personnels élaborés avec les intervenants. Les services sont offerts de façon continue et intensive afin de faciliter le processus de rétablissement et maintenir la continuité des soins (MSSLD, 1998b). Les personnes sont référées par les centres de santé, par leur médecin ou par leur famille, ou elles réclament d'elles-mêmes les services.

\section{Les préparatifs à la supervision}

Les stagiaires que je supervise sont inscrits en première ou en deuxième année au programme de maitrise de l'École de service social de l'Université d'Ottawa. La durée du stage est de 3 mois.

Chaque année, l'école invite les superviseurs de stages à présenter leurs milieux de travail aux étudiantes et aux étudiants et de leur donner un aperçu des attentes et des objectifs des stages. Cette initiative permet à ces derniers d'explorer diverses possibilités de stage et d'établir un contact avec les superviseurs.

La coordonnatrice de stage de l'école apporte une aide indispensable pour jumeler les stagiaires à un milieu répondant à leurs intérêts et aptitudes. Je rencontre alors l'étudiante ou l'étudiant qui m'est confié afin de discuter, entre autres, de mes attentes, de ses expériences professionnelles antérieures et de notre mode de fonctionnement. Je prépare un cartable avec des 
informations sur le fonctionnement de l'organisation et du travail quotidien, sur les lois en santé mentale et les recherches effectuées sur les ÉCTI.

Tout au long du stage, je maintiens un contact régulier pour vérifier et évaluer la quantité et qualité de travail effectué. Je demeure disponible en tout temps pour répondre aux questions, recevoir des commentaires ou résoudre des difficultés afin de réduire toute forme de stress créé par la nouveauté ou l'inexpérience. Tout en m'assurant que le rythme du travail et des apprentissages exigés conviennent à la stagiaire ou au stagiaire, je me garde de créer des illusions, puisque le travail social reste sans contredit exigeant et rempli de défis.

\section{Contrat de stage}

Dès la première semaine,j'invite l'étudiante ou l'étudiant à rédiger les objectifs de son contrat d'apprentissage. Le début du stage porte sur son orientation, notamment dans le contexte du milieu du stage, le fonctionnement de l'équipe et la population bénéficiaire de ses services. J'apprends à connaître sa façon de faire, sa manière d'être et je tente d'établir un climat de confiance et d'échange propice à l'évolution des apprentissages. À ce stade, l'étudiante ou l'étudiant consacre son temps notamment à observer, à lire, à consulter les dossiers, à m'accompagner lors de mes visites en communauté et à accompagner d'autres intervenants afin de mieux comprendre le rôle de chacun. Je l'encourage à écrire ses observations, réflexions ou questions dans un journal de bord auquel se référer lors des rencontres de supervision. De plus, la stagiaire ou le stagiaire assiste aux réunions quotidiennes, apprend à écrire des notes évolutives et commence à saisir notre mode de fonctionnement.

À la mi-stage, je révise les objectifs de son contrat et nous discutons de ses apprentissages tant sur le plan de son savoir-faire que sur celui de son savoir-être. Nous modifions la charge de travail si nécessaire. Ce retour permet de discuter et d'inclure des éléments théoriques en lien avec la pratique. Lorsque la stagiaire 
ou le stagiaire commence à diriger des entrevues, les périodes de supervision sont consacrées aux échanges sur ses apprentissages, ses réflexions ou aux enjeux rencontrés lors de ses interventions. Lors des dernières semaines du stage, l'étudiante ou l'étudiant effectue seul des visites de suivi, raffine son savoir-être et son savoir-faire en matière d'intervention et de rédige ses propres notes d'évolution. La rétroaction se fait de façon continue, sans oublier les appréciations sur les bons coups observés en intervention. À la fin du stage, le contrat initial sert d'outil de référence pour rédiger une évaluation synthèse et appuyer une réflexion sur sa réussite.

\section{Défis du milieu de stage}

Les stagiaires font face à plusieurs défis, dont certains relèvent du fonctionnement de l'équipe. Comme mentionné,l'ÉCTI est une équipe interdisciplinaire, mobile, composée de divers professionnels de la santé mentale qui se déplacent dans la communauté pour offrir ses services aux personnes vivant avec des problèmes sévères et persistants de santé mentale. C'est l'équipe entière qui assume la responsabilité d'aider le client à satisfaire ses besoins dans les diverses sphères de la vie en prodiguant des services spécialisés et individualisés de traitement, de réadaptation ou de soutien (Brouillette, l'Abbé et Tremblay, 2002; Allness et Knoedler, 1999; Test, 1998; Gélinas, 2002). Il est donc essentiel pour les stagiaires d'apprendre à travailler en équipe, à entendre des opinions diverses et à exprimer les leurs pendant les discussions cliniques.

Contrairement à l'approche de gestion de cas, tous les membres de l'ÉCTI connaissent tous les bénéficiaires de ses services, vu qu'ils partagent les responsabilités d'offrir à ces personnes un maximum de soutien. Les stagiaires doivent donc apprendre à partager leur rôle et à faire confiance aux autres membres de l'équipe pour assurer le suivi et assumer des tâches qui ne font pas traditionnellement partie de celles qui incombent au service social. Par exemple, des stagiaires pourraient accompagner un client dans une recherche de logement, à un rendez-vous médical ou encore 
à une activité physique. Ces mêmes tâches peuvent ensuite être confiées à d'autres intervenants lors de visites ultérieures.

Un autre défi rencontré dans ce stage est d'apprendre à intervenir tout en conduisant en voiture. Nous accompagnons les clients à des rendez-vous médicaux et communautaires et nous devons être en mesure de profiter de ce temps qui se prête souvent à la discussion de sujets problématiques ou à des confidences.

Comme nous travaillons avec des personnes qui vivent avec un lourd passé psychiatrique, nous sommes appelés à transiger avec des situations de crise qui peuvent survenir à tout moment durant la journée. Cela nous oblige à cerner nos priorités et à réviser notre horaire pour répondre au besoin immédiat. Nous devons nous montrer flexibles, transparents et prêts à intervenir dans toute situation imprévisible, ce qui peut parfois insécuriser les stagiaires.

Nous avons mentionné précédemment que le programme ÉCTI privilégie la réadaptation psychosociale. Il va sans dire que l'objectif pour une personne de retourner sur le marché du travail, par exemple, peut entraîner des démarches longues et ardues. La patience et la persévérance deviennent des vertus à développer. Parmi les difficultés que doivent surmonter nos bénéficiaires, mentionnons la peur de ne pas être à la hauteur, la stigmatisation, l'adaptation à un nouvel environnement et des rechutes. Cependant, chacun a ses forces, ses capacités, ses talents et c'est en leur donnant l'occasion de les développer que l'on découvre à quel point les personnes atteintes de maladie mentale sont capables de résilience et de survivre dans des contextes socioéconomiques précaires, souvent au-delà de ce que nous pouvons imager.Les stagiaires ont comme défi de développer leur sensibilité envers les facteurs contextuels et leurs impacts sur la santé mentale. La réadaptation n'est pas linéaire, mais parsemée d'essais et erreurs. Ainsi, en plus d'acquérir des connaissances sur les complexités de la santé mentale, les stagiaires doivent raffiner leur savoir-être pour faire preuve de grande empathie et de compréhension du point de vue de l'autre et accepter de suivre le rythme de la personne. 
"L'approche ACT est basée sur le fait que tous les citoyens incluant ceux qui sont touchés par les maladies mentales les plus graves et persistantes ont le droit de vivre une vie décente et satisfaisante dans la communauté » (Test, 1998, dans Gélinas, 2002, p. 4).Ainsi, semer l'espoir et soutenir la personne à atteindre ses buts sont des éléments essentiels pour activer le processus de réadaptation psychosociale. Être sensible à la signification que les personnes donnent à leurs trajectoires de vie constitue un apprentissage essentiel et parfois difficile pour les stagiaires. Ils doivent donc s'ouvrir à l'idée de se questionner sur des enjeux éthiques et de confronter les idées qu'ils se font des personnes auprès de qui ils interviennent. Il s'agit d'un travail essentiel devant être intégré aux discussions de la supervision.

Pour conclure, la supervision des étudiantes et des étudiants est une expérience enrichissante qui exige une capacité de remise en question de la part des superviseurs. Identifier nos forces et nos lacunes permet d'être honnête envers nous-mêmes et envers nos stagiaires. Michaud (1995) souligne l'importance de se comprendre soi-même quand on veut comprendre d'autres personnes et bien voir à leurs besoins. Chaque stagiaire m'a apporté sa façon de faire et ses acquis et avec ce tout, nous avons appris à travailler ensemble. L'expérience de superviser des stagiaires est valorisante et enrichissante tant au niveau professionnel que personnel. Ces étudiantes et étudiants me font réfléchir sur mon travail et m'amènent à m'interroger davantage sur mes interventions auprès des personnes de même que sur l'impact de mes décisions sur la vie de ces dernières.

\section{Bibliographie}

AGENCE DE LA SANTÉ PUBLIQUE DU CANADA (2002). Examen des meilleures pratiques de la réforme des soins de la santé mentale, réf. du 3 mars 2014, http://www.phac-aspc.gc.ca/mh-sm/ pubs/disc_paper/index-fra.php

ALLNESS, Deborah J., et William H. KNOEDLER (1999). The PACT Model of Community-Based Treatment for Persons with Severe and Persistent Mental Illnesses: A manual for PACT Start-up.

ALLNESS, Deborah J., et William H. KNOEDLER (2003). The National Program Standards for ACT

Teams,réf. Du 3 mars 2014, http://www.magellanofpa.com/media/700961/assertive_community_ treatment_act_national_program_standards_oct_2013.pdf 
BROUILLETTE, Carl, Bernard L'ABBÉ et Manon TREMBLAY (2002). «Équipes communautaires de traitement intensif: description et expériences des équipes parrainées par l'Hôpital Montfort ", Reflets : revue d'intervention sociale et communautaire, Vol. 8, № 1, p.114-124.

GÉLINAS, Daniel (2002). "Le suivi intensif en équipe dans la communauté et la réadaptation psychosociale font-ils bon ménage? ».Allocution prononcée à Montréal dans le cadre du colloque Suivi intensif en équipe dans la communauté : Fidélité au modèle ACT et stratégie d'implantation organisé par l'Association des hôpitaux du Québec le 21 novembre 2002.

LAFAVE, Hugh G., Henry R. DE SOUSA et Gary J. GERBER (1996). "Assertive Community Treatment of Severe Mental Illness: A Canadian Experience ", Psychiatric Services, Vol. 47 № 7 , p. 757-759.

MICHAUD, Pierre (1995). La supervision pédagogique, réflexion théorique et exercices pratiques, cahier 1, [notes de cours], Ottawa, Université d'Ottawa.

MINISTĖRE DE LA SANTÉ ET DES SOINS DE LONGUE DURÉE (1998a). Franchir les étapes : Cadres de prestation des services de santé mentale et des services de soutien connexes, réf. du 3 mars 2014, http://www.health.gov.on.ca/fr/public/publications/mental/docs/makehap_framework.pdf

MINISTÈRE DE LA SANTÉ ET DES SOINS DE LONGUE DURÉE (1998b). Standards for assertive community treatment teams, réf. du 3 mars 2014, http://www.health.gov.on.ca/en/ common/ministry/publications/reports/mentalhealth/act_standards.pdf

MINISTÈRE DE LA SANTÉ ET DES SOINS DE LONGUE DURÉE (2012). Ontario ACT team Directory 2012, Key Contacts for Ontario's Assertive Community Treatment teams.

TEST, Marianne (1998). «Modèles de traitement dans la communauté pour adultes ayant des maladies mentales graves et persistantes ", Santé mentale du Québec, Vol. 23, № 2, p.119-147. 